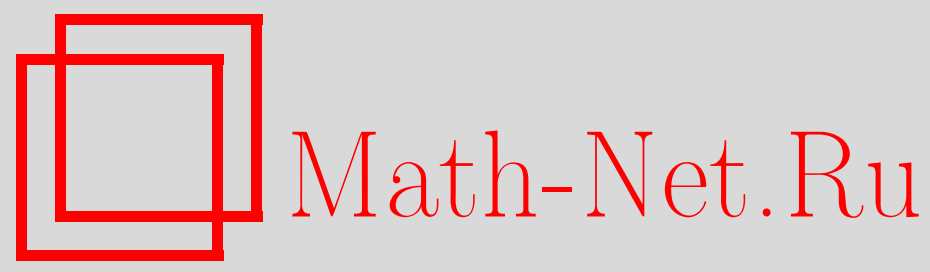

Л. Ю. Глебский, Локальные линейные операторы и многообходные решения однородных решеток связанных отображений, Матем. заметки, 1999, том 65, выпуск 1, 37-47

DOI: https://doi.org/10.4213/mzm1026

Использование Общероссийского математического портала Math-Net.Ru подразумевает, что вы прочитали и согласны с пользовательским соглашением http://www.mathnet.ru/rus/agreement

Параметры загрузки:

IP : 18.234 .197 .8

26 апреля 2023 г., 12:40:18

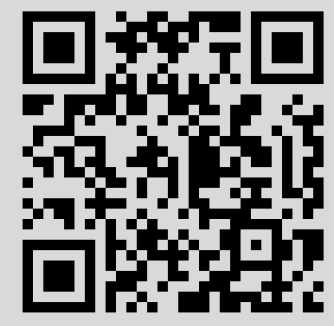




\title{
ЛОКАЛЬНЫЕ ЛИНЕЙНЫЕ ОПЕРАТОРЫ \\ И МНОГООБХОДНЫЕ РЕШЕНИЯ \\ ОДНОРОДНЫХ РЕШЕТОК СВЯЗАННЫХ ОТОБРАЖЕНИЙ
}

\author{
Л. Ю. Глебский
}

Доказаны теоремы о связи спектров линейньх операторов вида $A+\sum g_{i} B_{i} g_{i}^{-1}$ и $A+B_{i}$, где $g_{i} \in G$, a $G$ - группа, действующая линейными изометрическими операторами. Предполагается, что замкнутые операторы $A, B_{i}$ обладают следующим свойством: $\left\|B_{i} A^{-1} g B_{j} A^{-1}\right\| \rightarrow 0$ при $d(e, g) \rightarrow \infty$. Здесь $d-$ левоинвариантная метрика на $G$ и $e$ - единица $G$. Кроме того, оператор $A$ инвариантен относительно действия группы $G$. Эти теоремы применяются для доказательства существования многообходных решений динамических систем на решетках.

Библиография: 5 названий.

1. $(A, G)$-локальные наборы линейных операторов. Пусть $\mathscr{B}$ - банахово пространство. Для краткости будем говорить “ $X$ - замкнутый оператор" вместо " $X$ - замкнутый линейный оператор на $\mathscr{B}$ с плотной областью определения”. В этой статье мы будем предполагать, что:

а) групша $G$ действует на $\mathscr{B}$ линейньми изометрическими операторами;

б) $A$ - замкнутьй $G$-инвариантный оператор на $\mathscr{B}$, т.е. $A=g A g^{-1}$ для всех $g \in G$;

в) $d(\cdot, \cdot)$ - левоинвариантная метрика на $G$, т.е. $d\left(h g_{1}, h g_{2}\right)=d\left(g_{1}, g_{2}\right)$ для любых $g_{1}, g_{2}, h \in G$.

Обозначим через $\rho(X) \subset \mathbb{C}$-резольвентноемножество оператора $X$, через $D(X) \subset \mathscr{B}-$ область определения $X$; через $\mathscr{L}(\mathscr{B})$ - множество ограниченных всюду определенных линейных операторов на $\mathscr{B} ; A_{\lambda}=A-\lambda E$, где $E$ - единичньй оператор на $\mathscr{B} ; \bar{g}=\left(g_{1}, \ldots\right.$, $\left.g_{n}\right) \in G^{n}$ и $d(\bar{g})=\inf _{j \neq k} d\left(g_{j}, g_{k}\right)$.

ОПрЕДЕЛЕНИЕ 1. Конечный набор замкнутых линейных операторов $\left\{X_{k}, k=1,2\right.$, $\ldots, n<\infty\}$ (возможно $X_{j}=X_{k}$ для $j \neq k$ ) называется $(A, G)$-локальным. если $D\left(X_{k}\right) \supset D(A)$ и для любого $\lambda \in \rho(A)$, любых $j \neq k$ выполняется $\left\|X_{k} A_{\lambda}^{-1} g X_{j} A_{\lambda}^{-1}\right\| \rightarrow 0$ при $d(e, g) \rightarrow \infty$, где $e, g \in G$ и $e-$ единища $G$. (Напомним, что $A$ предполагается $G$-инвариантньм.)

Работа выполнена при поддержке Российского фонда фундаментальных исследований, гранты № 98-01-00790 и № 98-01-00795. 
TеОрема 1. Пусть $A$-замкнутый $G$-инвариантный оператор на $\mathscr{B} u\left\{B_{k}, k=\right.$ $1, \ldots, n\}$ есть $(A, G)$-локальный набор операторов. Тогда для любого $\lambda \in \rho(A) \cap$ $\bigcap_{k=1}^{n} \rho\left(A+B_{k}\right)$ существует $C>0$ такое, что

$$
\lambda \in \rho\left(A+\sum_{k=1}^{n} g_{k} B_{k} g_{k}^{-1}\right)
$$

nри $d(\bar{g})>$ C. Кроме того,

$$
\left\|\left(A_{\lambda}+\sum g_{k} B_{k} g_{k}^{-1}\right)^{-1}-\left(A_{\lambda}^{-1}+\sum_{k=1}^{n} g_{k}\left(\left(A_{\lambda}+B_{k}\right)^{-1}-A_{\lambda}^{-1}\right) g_{k}^{-1}\right)\right\| \rightarrow 0
$$

$n p u d(\bar{g}) \rightarrow \infty$.

Прежде чем доказывать теорему, сформулируем одно следствие. Пусть $A$ и $B_{k}$ удовлетворяют условию теоремы 1 . Обозначим через $S, S \subset \mathbb{C}$, ограниченное открытое множество, граница которого $\partial S$ есть замкнутая Жорданова кривая такая, что $\partial S \subset$ $\rho(A) \cap \bigcap_{k=1}^{n} \rho\left(A+B_{k}\right)$. Определены следуюшие проекторы:

$$
P_{0}=-\frac{1}{2 \pi i} \oint_{\partial S} A_{\lambda}^{-1} d \lambda, \quad P_{k}=-\frac{1}{2 \pi i} \oint_{\partial S}\left(A_{\lambda}+B_{k}\right)^{-1} d \lambda .
$$

В силу компактности $\partial S$ и теоремы 1 для достаточно больших $d(\bar{g})$ также определен проектор

$$
\Pi=-\frac{1}{2 \pi i} \oint_{\partial S}\left(A_{\lambda}+\sum_{k=1}^{n} g_{k} B_{k} g_{k}^{-1}\right)^{-1} d \lambda .
$$

СледСтвиЕ 1. Для проекторов $P_{k}, \Pi$, определенных формулами (1), (2), существуют следующие предель:

$$
\begin{gathered}
\left\|\left(P_{k}-P_{0}\right) g\left(P_{j}-P_{0}\right)\right\| \rightarrow 0 \quad \text { nри } \quad d(e, g) \rightarrow \infty \quad \text { для всех } \quad k \neq j, \\
\left\|\Pi-\left(P_{0}+\sum_{k=1}^{n} g_{k}\left(P_{k}-P_{0}\right) g_{k}^{-1}\right)\right\| \rightarrow 0 \quad n p u \quad d(\bar{g}) \rightarrow \infty .
\end{gathered}
$$

ДоКАЗАТЕЛЬСТво ТЕОРЕмЫ 1 . Доказательство теоремы основано на следующем тождестве:

$$
\begin{gathered}
\left(A_{\lambda}+\sum_{k=1}^{n} g_{k} B_{k} g_{k}^{-1}\right)\left(A_{\lambda}^{-1}+\sum_{k=1}^{n} g_{k}\left(\left(A_{\lambda}+B_{k}\right)^{-1}-A_{\lambda}^{-1}\right) g_{k}^{-1}\right) \\
=E+\sum_{j \neq k} g_{j} B_{j} g_{j}^{-1} g_{k}\left(\left(A_{\lambda}+B_{k}\right)^{-1}-A_{\lambda}^{-1}\right) g_{k}^{-1}=E+\delta
\end{gathered}
$$

здесь использовано равенство $A_{\lambda}+g_{k} B_{k} g_{k}^{-1}=g_{k}\left(A_{\lambda}+B_{k}\right) g_{k}^{-1}$. Преобразовав $\delta$ :

$$
\delta=-\sum_{j \neq k} g_{j} B_{j} A_{\lambda}^{-1} g_{j}^{-1} g_{k} B_{k} A_{\lambda}^{-1} A_{\lambda}\left(A_{\lambda}+B_{k}\right)^{-1} g_{k}^{-1},
$$


получаем, что $\|\delta\| \rightarrow 0$ при $d(\bar{g}) \rightarrow \infty$ по определению $(A, G)$-локального набора операторов. Таким образом, оператор $A_{\lambda}+B(\bar{g})=A_{\lambda}+\sum_{k=1}^{n} g_{k} B_{k} g_{k}^{-1}$ имеет правьй обратньй, если $d(\bar{g})$ достаточно велико. Аналогичные вычисления показьвают, что $E+B(\bar{g}) A_{\lambda}^{-1}$ (а значит, и $\left.A_{\lambda}+B(\bar{g})\right)$ имеет левьй обратньй для достаточно больших $d(\bar{g})$ :

$$
\left(E+\sum_{k=1}^{n} g_{k}\left(\left(E+B_{k} A_{\lambda}^{-1}\right)^{-1}-E\right) g_{k}^{-1}\right)\left(E+\sum_{k=1}^{n} g_{k} B_{k} A_{\lambda}^{-1} g_{k}^{-1}\right)=E+\delta^{\prime}
$$

где $\left\|\delta^{\prime}\right\| \rightarrow 0$ при $d(\bar{g}) \rightarrow \infty$. Таким образом,

$$
\left(A_{\lambda}+\sum_{k=1}^{n} g_{k} B_{k} g_{k}^{-1}\right)^{-1}=\left(A_{\lambda}^{-1}+\sum_{k=1}^{n} g_{k}\left(\left(A_{\lambda}+B_{k}\right)^{-1}-A_{\lambda}^{-1}\right) g_{k}^{-1}\right)(E+\delta)^{-1}
$$

что доказьвает теорему 1.

ДокАЗАТЕЛЬСТво СЛЕДСТВИя 1. Для доказательства предельного соотношения (4) достаточно проинтегрировать выражение (5) по кривой $\partial S$. Для доказательства (3) возьмем $S^{\prime} \subset S$ такое, что $\left(S-S^{\prime}\right) \in \rho\left(A+B_{k}\right) \cap \rho\left(A+B_{j}\right), \partial S^{\prime}$ есть замкнутая спрямляемая кривая и $\partial S^{\prime} \cap \partial S=\varnothing$. Тогда

$$
\begin{aligned}
\left(P_{k}\right. & \left.-P_{0}\right) g\left(P_{j}-P_{0}\right) \\
& =-\frac{1}{4 \pi^{2}} \oint_{\partial S} \oint_{\partial S^{\prime}}\left(\left(A_{\lambda}+B_{k}\right)^{-1}-A_{\lambda}^{-1}\right) g\left(\left(A_{\mu}+B_{j}\right)^{-1}-A_{\mu}^{-1}\right) d \lambda d \mu \\
& =-\frac{1}{4 \pi^{2}} \oint_{\partial S} \oint_{\partial S^{\prime}}\left(A_{\lambda}+B_{k}\right)^{-1} B_{k} \frac{\left(A_{\lambda}^{-1}-A_{\mu}^{-1}\right)}{\mu-\lambda} g B_{j}\left(A_{\mu}+B_{j}\right)^{-1} d \lambda d \mu \rightarrow 0
\end{aligned}
$$

при $d(e, g) \rightarrow \infty$.

Для оператора $X$ положим $\operatorname{dim} X=\operatorname{dim} \operatorname{Im} X$ (размерность оператора по определению есть размерность его образа). Верно следующее

ПРЕДЛОЖЕНИЕ 1. Пусть П, $P_{i} \in \mathscr{L}(\mathscr{B}), i=1, \ldots, n,-$ проекторы,$\Delta=\Pi-\sum_{i=1}^{n} P_{i}$. Предположим, что для всех $1 \leqslant i, j \leqslant n, i \neq j$, выполняется $(n-1)\left\|P_{j} P_{i}\right\|+$ $n\left\|P_{i} \Delta\right\|<1$. Тогда $\operatorname{dim} \Pi=\sum_{i=1}^{n} \operatorname{dim} P_{i}$.

Из предложения 1, теоремы 1 и следствия 1 вытекает

СлЕДСтВИЕ 2. Пусть $D(B) \supset D(A)$ и для всех $\lambda \in \rho(A)$ существует предел $\left\|B A_{\lambda}^{-1} g B A_{\lambda}^{-1}\right\| \rightarrow 0$ nри $d(e, g) \rightarrow \infty$. Предположим, что $\lambda_{0} \in \rho(A)-$ изолированная спектральная точка кратности $l$ оператора $A+B$. Тогда $\lambda_{0}$ имеет окрестность $S \ni \lambda_{0}$ такую, что для каждого $n \in \mathbb{N}$ найдется $C>0$ такое, что $S$ содержит в точности $l \cdot n$ точек спектра оператора

$$
A+\sum_{k=1}^{n} g_{k} B g_{k}^{-1}, \quad g_{k} \in G, \quad d(\bar{g})>C
$$

спектральные точки считаются с кратностью. 
ПРимеР. Рассмотрим уравнение Шрёдингера

$$
-\Delta \xi+\sum_{i} U\left(x+h_{i}\right) \xi=\lambda \xi
$$

в $L_{2}\left(\mathbb{R}^{k}, \mathbb{C}\right)$, где $x \in \mathbb{R}^{k}, \Delta=\sum \partial_{x_{k}}^{2}$ и $h_{i} \in \mathbb{R}^{k}$. Пусть групша $G=\left(\mathbb{R}^{k},+\right)$ с $d\left(g_{1}, g_{2}\right)=$ $\left|g_{1}-g_{2}\right|$. Тогда верно следуюшее

ПРЕДЛОЖЕНИЕ 2. Если $U(\cdot) \in L_{\infty}\left(\mathbb{R}^{k}, \mathbb{C}\right)$ u $U(x) \rightarrow 0$ npu $|x| \rightarrow \infty$, то набор $\left\{B_{k}=U, k=1, \ldots n\right\} \quad(\Delta, G)$-локален в $L_{2}\left(\mathbb{R}^{k}, \mathbb{C}\right)$.

ДокАЗАтЕльство. Пусть оператор $K: L_{2} \rightarrow L_{2}$ определен соотношением

$$
(K \xi)(x)=\int_{y} \widehat{K}(x, y) \xi(y) d y, \quad \text { где } \widehat{K}(\cdot, \cdot): \mathbb{R}^{k} \times \mathbb{R}^{k} \rightarrow \mathbb{C} .
$$

Пусть $K=U \Delta_{\lambda}^{-1} g_{h} U \Delta_{\lambda}^{-1}$ или

$$
\widehat{K}_{h}(x, y)=\int_{z} U(x) \Gamma(x-z) U(z+h) \Gamma(z+h-y) d z,
$$

где $\Gamma$ - функция Грина оператора $\Delta_{\lambda}$. Вычисления показывают, что

$$
\sup _{y} \int_{x}\left|\widehat{K}_{h}(x, y)\right| d x \leqslant C_{1}, \quad \sup _{x} \int_{y}\left|\widehat{K}_{h}(x, y)\right| d y \rightarrow 0 \quad \text { при } h \rightarrow \infty .
$$

Используя оценку

$$
\|K\|_{2} \leqslant\left(\sup _{x} \int_{y}|\widehat{K}(x, y)| d y \cdot \sup _{y} \int_{x}|\widehat{K}(x, y)| d x\right)^{1 / 2},
$$

получим $(\Delta, G)$-локальность набора $\left\{B_{k}=U, k=1, \ldots n\right\}$. Этот набор остается $(\Delta, G)$-локальным, если $G$ - группа движений $\mathbb{R}^{k}(\operatorname{coxраняющая~} \Delta)$.

2. Бесконечные $(A, G)$-локальные наборы. В этом пункте мы дадим вариант определения бесконечных $(A, G)$-локальных наборов линейных операторов. На банахово пространство $\mathscr{B}$, группу $G$ и оператор $A$ наложены те же условия, что и вьше.

ОПрЕДЕЛЕниЕ 2. Бесконечный набор замкнутых операторов $\left\{B_{j}, j=1,2, \ldots\right\}$, $D\left(B_{j}\right) \supset D(A)$, назьвается $(A, G)$-локальным, если для любого $\lambda \in \rho(A)$ операторы $T_{k}=B_{k} A_{\lambda}^{-1}$ обладают следуюшими свойствами:

1) существуют $C_{1}>0$ и $C_{2}>0$ такие, что для любых $x \in \mathscr{B}, \bar{g}=\left\langle g_{1}, g_{2}, \ldots\right\rangle$, $g_{i} \in G$ и $d(\bar{g})>C_{1}$ справедливо неравенство

$$
\sum_{k \in \mathbb{N}}\left\|g_{k} T_{k} g_{k}^{-1} x\right\| \leqslant C_{2}\|x\|
$$

2) существуют последовательность операторов $T_{k, l}$ и числовая последовательность $C_{l} \in \mathbb{R}^{+}$такие, что при $d(\bar{g})>C_{1}$ вьполняется

$$
\frac{\sum_{k \in \mathbb{N}}\left\|g_{k}\left(T_{k, l}-T_{k}\right) g_{k}^{-1} x\right\|}{\|x\|} \rightarrow 0 \quad \text { при } l \rightarrow \infty
$$

равномерно по $\bar{g}$ и $x \in \mathscr{B}, x \neq 0$, и $T_{k, l} g T_{j, l}=0$ при $d(e, g)>C_{l}$. 
Предположим, что $\rho(A) \neq \varnothing, \lambda \in \rho(A)$ и $\left\{B_{k}\right\}$ - бесконечньй набор $(A, G)$-локальньх операторов. Очевидно, что ряд $\sum g_{k} B_{k} A_{\lambda}^{-1} g_{k}^{-1}$ имеет сильный предел при достаточно больших $d(\bar{g})$ и, стало быть, определен оператор

$$
A+\sum g_{k} B_{k} g_{k}^{-1}=A+\left(\sum g_{k} B_{k} A_{\lambda}^{-1} g_{k}^{-1}\right) A_{\lambda} .
$$

TEOРема 2. Пусть $\left\{B_{k}, k=1,2, \ldots\right\}$ есть $(A, G)$-локальный набор операторов, $\lambda \in \rho(A) \cap \bigcap_{k=1}^{\infty} \rho\left(A+B_{k}\right) u\left\|\left(A_{\lambda}+B_{k}\right)^{-1}\right\|<M$ для всех $k$ и некоторого $M>0$. Тогда

1) $p_{я \partial}$

$$
\sum_{k \in \mathbb{N}} g_{k}\left(\left(A_{\lambda}+B_{k}\right)^{-1}-A_{\lambda}^{-1}\right) g_{k}^{-1}
$$

имеет сильный предел при достаточно больиих $d(\bar{g})$;

2) оператор

$$
A_{\lambda}+\sum_{k=1}^{n} g_{k} B_{k} g_{k}^{-1}
$$

обратим для любого $n \in \mathbb{N} \cup\{\infty\}$ при достаточно большом $d(\bar{g})$ и выполняется

$$
\begin{aligned}
& \left\|\left(A_{\lambda}+\sum_{k=1}^{n} g_{k} B_{k} g_{k}^{-1}\right)^{-1}-\left(A_{\lambda}^{-1}+\sum_{k=1}^{n} g_{k}\left(\left(A_{\lambda}+B_{k}\right)^{-1}-A_{\lambda}^{-1}\right) g_{k}^{-1}\right)\right\| \rightarrow 0 \\
& \quad \text { при } d(\bar{g}) \rightarrow \infty \text { равномерно по } n \in \mathbb{N} \cup\{\infty\} .
\end{aligned}
$$

ДокАЗАТЕЛЬСтво. Докажем сходимость ряда

$$
\begin{aligned}
\sum_{k=1}^{n}\left\|g_{k}\left(\left(A_{\lambda}+B_{k}\right)^{-1}-A_{\lambda}^{-1}\right) g_{k}^{-1} x\right\| & =\sum_{k=1}^{n}\left\|g_{k}\left(A_{\lambda}+B_{k}\right)^{-1} g_{k}^{-1} g_{k} B_{k} A_{\lambda}^{-1} g_{k}^{-1} x\right\| \\
& \leqslant M \sum_{k=1}^{n}\left\|g_{k} T_{k} g_{k}^{-1} x\right\| \leqslant M C_{1}
\end{aligned}
$$

где $T_{k}$ и $C_{1}$ взяты из определения 2.

Нам достаточно показать обратимость

$$
S(n)=E+\sum_{k=1}^{n} g_{k} B_{k} A_{\lambda}^{-1} g_{k}^{-1}=E+\sum_{k=1}^{n} g_{k} T_{k} g_{k}^{-1} .
$$

Пусть $S_{l}(n)=E+\sum_{k=1}^{n} g_{k} T_{k, l} g_{k}^{-1}$. Простым умножением убеждаемся, что

$$
S_{l}^{-1}(n)=E+\sum_{k=1}^{n} g_{k}\left(\left(E+T_{k, l}\right)^{-1}-E\right) g_{k}^{-1} .
$$

Последовательности операторов $S(n), S_{l}(n)$ и $S_{l}^{-1}(n)$ имеют сильные пределы при фиксированном $l$ и $n \rightarrow \infty$. Обозначим их через $S_{0}, S_{l, 0}$ и $K_{l, 0}$ соответственно. Стало быть, $S_{l, 0}^{-1}$ существует и $S_{l, 0}^{-1}=K_{l, 0}$. Кроме того, по определению 2 можно найти последовательность $\varepsilon_{l}>0$, сходящуюся к 0 , такую, что

$$
\left\|S_{0}-S_{l, 0}\right\|<\varepsilon_{l} \quad \text { и }\left\|S_{l, 0}^{-1}\right\|<\left(M+\varepsilon_{l}\right)\left(C_{1}+\varepsilon_{l}\right) .
$$

Таким образом, оператор $S_{0}^{-1}$ существует, если $\varepsilon_{l}\left(M+\varepsilon_{l}\right)\left(C_{1}(\lambda)+\varepsilon_{l}\right)<1$. Аналогично доказьвается утверждение 2) теоремы. 
3. Многообходные локализованные решения. Имея ввиду приложения к решеткам связанных отображений, предположим, что $\mathscr{B}=\mathscr{B}_{1}^{*}$, где $\mathscr{B}_{1}$ - банахово пространство, а $\mathscr{B}_{1}^{*}$ - банахово пространство линейных ограниченных функционалов на $\mathscr{B}_{1}$. Кроме того, будем предполагать, что

a) группа $G$ действует на $\mathscr{B}_{1}$ линейными изометрическими операторами;

б) $\mathscr{A}$ - замкнутый $G$-инвариантньй оператор на $\mathscr{B}_{1}$;

в) $d(\cdot, \cdot)$ - левоинвариантная метрика на $G$, т.е. $d\left(h g_{1}, h g_{2}\right)=d\left(g_{1}, g_{2}\right)$ для любых $g_{1}, g_{2}, h \in G$.

В этом случае на $\mathscr{B}$ определено сопряженное действие $G$ : для $x \in \mathscr{B} g x=\left(g^{-1}\right)^{*} x$, где элемент группы $G$ и его действие на $\mathscr{B}_{1}$ и $\mathscr{B}$ обозначено одной и той же буквой. При этом оператор $A=\mathscr{A}^{*}$ будет $G$-инвариантньм.

На $\mathscr{B}$ определена $w^{*}$-топология и пространство $\mathscr{B}$ полно относительно этой топологии (см. [1]).

Пусть $F: \mathscr{B} \rightarrow \mathscr{B}$ есть $G$-инвариантная $(g F(x)=F(g x))$ дважды непрерьвно дифференцируемая функция. В следующем определении бесконечные суммы понимаются как $w^{*}$-пределы соответствующих частных сумм.

ОПРЕДЕЛЕНИЕ 3 . Набор $\left\{x_{k} \in \mathscr{B}\right\}, k=1, \ldots, n, n \in \mathbb{N} \cup\{\infty\}$, называется $(F, G)$-лoкальным. если

1) $\left\|F\left(\sum_{k=1}^{m} g_{k} x_{k}\right)-\sum_{k=1}^{m} g_{k} F\left(x_{k}\right)\right\| \rightarrow 0$ при $d(\bar{g}) \rightarrow \infty$ равномерно по $m \leqslant n$;

2) $\left\|D F\left(\sum_{k=1}^{m} g_{k} x_{k}\right)-\sum_{k=1}^{m} g_{k} D F\left(x_{k}\right) g_{k}^{-1}\right\| \rightarrow 0$ при $d(\bar{g}) \rightarrow \infty$ равномерно по $m \leqslant n$ (так как $F G$-инвариантна, верно равенство $\left.D F(g x)=g D F(x) g^{-1}\right)$;

3) $\left\|D^{2} F\left(\xi+\sum_{k=1}^{m} g_{k} x_{k}\right)\right\|$ равномерно ограничено при $\|\xi\|<\varepsilon, d(\bar{g})>C, m \leqslant n$.

Если $m=\infty$, предполагается, что все ряды данного определения имеют $w^{*}$-предел при достаточно большом $d(\bar{g})$.

Пример такого набора описан ниже в лемме 2.

Рассмотрим уравнение

$$
F(x)=0 .
$$

Tеорема 3. Пусть $F: \mathscr{B} \rightarrow \mathscr{B}-C^{2}$-гладкая $G$-инвариантная функиия и $F(x)=$ $A x+\widetilde{F}(x)$, где $A=\mathscr{A}^{*} u \mathscr{A} \in \mathscr{L}\left(\mathscr{B}_{1}\right)$ является $G$-инвариантным. Предполохим, что $x_{k} \in \mathscr{B}, k=1, \ldots, n, n \in \mathbb{N} \cup\{\infty\},-$ корни уравнения (6) такие, что

1) набор әлементов $\left\{x_{k} \in \mathscr{B}\right\}$ является $(\widetilde{F}, G)$-локальным;

2) операторь $D \widetilde{F}\left(x_{k}\right)=B_{k}^{*}$ и набор операторов $\left\{B_{k}\right\}$ является $(\mathscr{A}, G)$-локальным в $\mathscr{B}_{1}$;

3) операторы $A^{-1},\left(A+D \widetilde{F}\left(x_{k}\right)\right)^{-1}$ существуют и ограничены равномерно по $k$ (если действие группь $G$ дифференцируемо, то такая ситуация можсет возникнуть только для дискретной группь $G$ ).

Тогда для любого достаточно малого $\varepsilon>0$ найдется $C>0$ такое, что для каждого $y=\sum_{k=1}^{m} g_{k} x_{k}, d(\bar{g})>C, m \leqslant n$, уравнение (6) имеет один и только один корень $x_{\bar{g}} \in\{x \in \mathscr{B},\|x-y\| \leqslant \varepsilon\}$.

ДокАЗАТЕльство. Если мы покажем существование и равномерную ограниченность по $\bar{g}, d(\bar{g})>C$, оператора $\left(A+\sum g_{k} D \widetilde{F}\left(x_{k}\right) g_{k}^{-1}\right)^{-1}$, то сможем применить теорему о неявной функции к уравнению

$$
\begin{aligned}
0=F(y+\xi)= & \left(A+\sum g_{k} D \widetilde{F}\left(x_{k}\right) g_{k}^{-1}\right) \xi+\left(\left(D \widetilde{F}\left(\sum g_{k} x_{k}\right)-\sum g_{k} D \widetilde{F}\left(x_{k}\right) g_{k}^{-1}\right) \xi\right. \\
& \left.+\left(F\left(\sum g_{k} x_{k}\right)-\sum g_{k} \widetilde{F}\left(x_{k}\right)\right)+O\left(\|\xi\|^{2}\right)\right)
\end{aligned}
$$


где $\left\|O\left(\|\xi\|^{2}\right)\right\|<K\|\xi\|^{2}$. Ввиду $(\widetilde{F}, G)$-локальности вторая и третья скобки в уравнении (7) малы равномерно по $\bar{g}, d(\bar{g})>C$. Нам осталось доказать существование и равномерную ограниченность по $\bar{g}, d(\bar{g})>C$, оператора $\left(A+\sum g_{k} D \widetilde{F}\left(x_{k}\right) g_{k}^{-1}\right)^{-1}$. Нам потребуется

ТЕОРемА 4 [1]. Замкнутый плотно определенный оператор $T$ на $\mathscr{B}_{1}$ обратим (имеет ограниченный обратный) тогда и только тогда, когда $T^{*}$ обратим. При әтом $\left(T^{-1}\right)^{*}=\left(T^{*}\right)^{-1} u\left\|T^{-1}\right\|=\left\|\left(T^{*}\right)^{-1}\right\|$.

Так как $A=\mathscr{A}^{*}$ и $D F\left(x_{k}\right)=B_{k}^{*}$, условие 3$)$ теоремы 3 эквивалентно тому, что операторы $\mathscr{A}^{-1},\left(\mathscr{A}+B_{k}\right)^{-1}$ существуют и ограничены. Далее, в силу $(\mathscr{A}, G)$-локальности набора $\left\{B_{k}\right\}$ получим существование и равномерную ограниченность по $d(\bar{g})$ оператора

$$
\left(\mathscr{A}_{\lambda^{*}}+\sum_{k} g_{k} B_{k} g_{k}^{-1}\right)^{-1} \text {. }
$$

Переходя к сопряженным операторам, получим требуемое утверждение.

4. Решетки связанных отображений. Рассмотрим приложение теоремы 3 к однородным решеткам связанных отображений (см. [2], [3]). Пусть $\ell_{\infty}=\left\{u_{\alpha} \in \mathbb{R}^{p}, \alpha \in \mathbb{Z}^{d}\right.$, $\left.\sup _{\alpha}\left|u_{\alpha}\right|<\infty\right\} \mathrm{c}\|u\|_{\infty}=\sup _{\alpha}\left|u_{\alpha}\right|$, где $\left|u_{\alpha}\right|=\left(u_{\alpha}, u_{\alpha}\right)^{1 / 2}$ для стандартного скалярного произведения $(\cdot, \cdot)$ в $\mathbb{R}^{p}$. Для $\beta \in \mathbb{Z}^{d}$ определим оператор сдвига $S_{\beta}$ на $\ell_{\infty}$ :

$$
\left(S_{\beta} u\right)_{\alpha}=u_{\alpha+\beta} \text {. }
$$

Пусть $\mathbb{Z}^{d} \supset I=\left\{i_{1}, i_{2}, \ldots, i_{r}\right\}$ - конечное множество. Для $u \in \ell_{\infty}$ обозначим

$$
\{\cdot\}_{I}: \ell_{\infty} \rightarrow \mathbb{R}^{p r}, \quad\{u\}_{I}=\left(u_{i_{1}}, u_{i_{2}}, \ldots, u_{i_{r}}\right) .
$$

ОПРЕДЕЛЕНИЕ 4 (см. [3]). Функция $F: \ell_{\infty} \rightarrow \ell_{\infty}$ называется однородной решеткой связанных отображений, если

$$
(F(u))_{\alpha}=f\left(\left\{S_{\alpha} u\right\}_{I}\right)
$$

для некоторых $I$ и $f: \mathbb{R}^{p r} \rightarrow \mathbb{R}^{p}$.

Справедливо следующее утверждение, связывающее гладкость $F$ и $f$.

ПрЕдЛОЖЕНИЕ 3. Функиия $F: \ell_{\infty} \rightarrow \ell_{\infty}$ является $C^{k}$-гладкой по Фреше тогда $u$ только тогда, когда функиия $f: \mathbb{R}^{p r} \rightarrow \mathbb{R}^{p} C^{k}$-гладкая.

Сейчас мы можем сформулировать теорему о существовании многообходных решений уравнений типа (6), когда $F$ - однородная решетка связанных отображений.

TеОрема 5. Пусть $F: \ell_{\infty} \rightarrow \ell_{\infty}$ есть $C^{2}$-гладкая однородная решетка связанных отобрахсений. Пусть $v \in \ell_{\infty}-$ корень уравнения (6). Предполохим, что $\left|v_{\alpha}\right| \rightarrow 0$ при $|\alpha| \rightarrow \infty$ и операторы $D F(0), D F(v)$ имеют ограниченные обратные. Tогда

1) ряд $\sum_{k=1}^{\infty} S_{\beta_{k}} v$ uмеет $w^{*}$-предел, если $\beta_{j} \neq \beta_{k}$ nрu $j \neq k$;

2) для любого достаточно малого $\varepsilon>0$ найдется $C>0$ такое, что для каждого $u=\sum_{k=1}^{n} S_{\beta_{k}} v, n \leqslant \infty,\left|\beta_{j}-\beta_{k}\right|>C$ при $j \neq k$, уравнение (6) имеет единственный корень $x_{\bar{\beta}}$ в $\varepsilon$-окрестности и. 
ЗАмЕчАниЕ. Мы назовем решение $x_{\bar{\beta}}$ многообходным. Из условий теоремы следует, что $F(0)=0$.

Прежде чем доказьвать теорему, рассмотрим подробнее случай,когда $d=1$ и $(F(u))_{j}$ $=u_{j+1}-g\left(u_{j}\right)$. В этом случае решения уравнения $(6)$ - траектории отображения $g$. Сейчас мы сформулируем условия на отображение $g$, эквивалентные условию теоремы 5 . Справедливость этой эквивалентности следует из предложений $4-6$, приведенных ниже.

Обозначим через $0_{\infty}, 0_{p}$ нулевые векторы в $\ell_{\infty}, \mathbb{R}^{p}$ соответственно. Так как $F\left(0_{\infty}\right)=$ $0_{\infty}$, то $g\left(0_{p}\right)=0_{p}$, т.е. $0_{p}-$ неподвижная точка отображения $g$. Если $g: \mathbb{R}^{p} \rightarrow \mathbb{R}^{p}-$ диффеоморфизм, то условия теоремы 5 эквивалентны тому, что $0_{p}$ - седло диффеоморфизма $g$, а его устойчивое и неустойчивое многообразия пересекаются трансверсально по траектории $v_{j}$.

Более того, оказьвается, что условия теоремы 5 можно перевести на динамический язык и в случае, когда $g$ не имеет обратного. Тогда обратимость $D F\left(0_{\infty}\right)$ эквивалентна тому, что матрица $D g\left(0_{p}\right)$ не имеет спектра на единичной окружности. В этом случае неподвижная точка $0_{p}$ имеет локальные многообразия: устойчивое $W^{s}\left(0_{p}\right)$ и неустойчивое $W^{u}\left(0_{p}\right), \operatorname{dim} W^{s}+\operatorname{dim} W^{u}=p\left(\right.$ см. [4]). Пусть $v_{j}-$ траектория из теоремы 5. Тогда $v_{j} \in W^{s}\left(0_{p}\right)$ при $j>M_{1}$ и $v_{j} \in W^{u}\left(0_{p}\right)$ при $j<M_{2}$. Обозначим через $T_{j}^{u}$ касательное к $W^{u}\left(0_{p}\right)$ пространство в точке $v_{j}$ (для $j<M_{2}$ ), а через $T_{j}^{s}$ касательное к $W^{s}\left(0_{p}\right)$ пространство в точке $v_{j}$ (для $j>M_{1}$ ). Мы можем определить $T_{j}^{s}$ и $T_{j}^{u}$ для всех $j \in \mathbb{Z}$, используя соотношения $T_{j+1}^{u}=D g\left(v_{j}\right) T_{j}^{u}$ и $T_{j}^{s}=\left(D g\left(v_{j}\right)\right)^{-1} T_{j+1}^{s}$; здесь $A^{-1} T=\{x: A x \in T\}$. Обратимость $D F(v)$ эквивалентна тому, что $T_{j}^{s} \cap T_{j}^{u}=\{0\}$ и $\operatorname{dim} T_{j}^{u}=\operatorname{dim} W^{u}\left(0_{p}\right)$ хотя бы для одного $j$ (а значит, и для всех $\left.j \in \mathbb{Z}\right)$.

Сейчас мы приведем ряд утверждений о линейных операторах, задаваемых равенствами

$$
\left(L_{0} \xi\right)_{j}=\xi_{j+1}-A \xi_{j}, \quad(L \xi)_{j}=\xi_{j+1}-A_{j} \xi_{j}
$$

где $A=D g(0), A_{j}=D g\left(v_{j}\right)$. Доказательства этих утверждений стандартны в теории динамических систем (см. [5]).

ПРЕДЛОЖЕНИЕ 4. Оператор $L_{0} \in \mathscr{L}\left(\ell_{\infty}\right)$ обратим тогда и только тогда, когда $A$ не имеет точек спектра на единичной окружности.

Пусть $A=A^{s} \oplus A^{u}$, где спектр $A^{s}\left(A^{u}\right)$ лежит внутри (вне) единичного круга. Разложению $A=A^{s} \oplus A^{u}$ соответствует разложение $\mathbb{R}^{p}=E^{s} \oplus E^{u}$.

ПредЛОЖЕНИЕ 5. Пусть $A_{j} \rightarrow A=A^{s} \oplus A^{u}$ npu $|j| \rightarrow \infty$. Тогда для любого $\lambda_{*}$, $\max \left\{|\lambda|: \lambda \in \operatorname{Sp}\left(A^{s}\right) \cup \operatorname{Sp}\left(\left(A^{u}\right)^{-1}\right)\right\}<\lambda_{*}<1$,

1) найдутся $M \in \mathbb{Z}^{+}, C>0$ и последовательность подпространств $E_{j}^{s} \subset \mathbb{R}^{p}$, $j=M, M+1, \ldots, E_{j}^{s}=\left\{x: A_{j} x \in E_{j+1}^{s}\right\}$, maкuе, ито $\operatorname{dim}\left(E_{j}^{s}\right)=\operatorname{dim}\left(E^{s}\right)$ и для любой последовательности векторов $x_{j} \in E_{j}^{s}, x_{j+1}=A_{j} x_{j}$, верна оценка $\left|x_{j+k}\right| \leqslant C \lambda_{*}^{k}\left|x_{j}\right|$, где $j, k \in \mathbb{Z}^{+} u j>M$;

2) найдутся $M \in \mathbb{Z}^{-}, C>0$ и последовательность подпространств $E_{j}^{u} \subset \mathbb{R}^{p}$, $j=M, M-1, \ldots, E_{j+1}^{u}=A_{j} E_{j}^{u}$, maкие, что $\operatorname{dim}\left(E_{j}^{u}\right)=\operatorname{dim}\left(E^{u}\right) u$ для любой последовательности векторов $x_{j} \in E_{j}^{u}, x_{j+1}=A_{j} x_{j}$, верна оценка $\left|x_{j-k}\right| \leqslant C \lambda_{*}^{k}\left|x_{j}\right|$, әде $k \in \mathbb{Z}^{+} u j<M$, а следовательно, существуют обратные операторы для ограничения $A_{j}$ на $E_{j}^{u},\left(A_{j} \mid E_{j}^{u}\right)^{-1} \in \mathscr{L}\left(E_{j+1}^{u}, E_{j}^{u}\right)$.

Можно определить $E_{j}^{s}$ и $E_{j}^{u}$ для всех $j \in \mathbb{Z}$, используя соотношения $E_{j+1}^{u}=A_{j} E_{j}^{u}$, $E_{j-1}^{s}=\left\{x \in \mathbb{R}^{p}: A_{j-1} x \in E_{j}^{s}\right\}$. 
ПРЕДЛОЖенИе 6. Оператор $L \in \mathscr{L}\left(\ell_{\infty}\right),(L \xi)_{j}=\xi_{j+1}-A_{j} \xi_{j}$, обратим тогда и только тогда, когда для некоторого $j \in \mathbb{Z}$ выполняется $E_{j}^{s} \cap E_{j}^{u}=\{0\}$ u $\operatorname{dim}\left(E_{j}^{u}\right)=\operatorname{dim}\left(E^{u}\right)$.

ДоКАЗАТЕЛЬСТВо ТЕОремЫ 5. Представим $F=A+\widetilde{F}$, где $A \in \mathscr{L}\left(\ell_{\infty}\right)$ и $D \widetilde{F}(0)=0$. Из лемм 1,2 , сформулированных ниже, следует, что набор $\left\{v_{k}=v, k=1,2, \ldots\right\}$ является $(\widetilde{F}, G)$-локальным, а также существуют операторы $\mathscr{A}$ и $B$ такие, что $A=\mathscr{A}^{*}$ и $D \widetilde{F}(v)=B^{*}$, а набор $\left\{B_{k}=B, k=1, \ldots\right\}$ является $(\mathscr{A}, G)$-локальным. Затем применим теорему 3 .

Напомним, что $\ell_{\infty}=\ell_{1}^{*}$, где $\ell_{1}=\left\{u_{\alpha} \in \mathbb{R}^{p}, \sum_{\alpha \in \mathbb{Z}^{d}}\left|u_{\alpha}\right|<\infty\right\}$.

Лемма 1. Пусть $F \in C^{2}\left(\ell_{\infty}, \ell_{\infty}\right)$ - однородная решетка связанных отображений. Предположим, ито $F(0)=0, F(v)=0,\left|v_{\alpha}\right| \rightarrow 0$ nрu $|\alpha| \rightarrow \infty u$ u $F(0), D F(v)$ имеют ограниченные обратные операторы. Тогда $v \in \ell_{1}$.

ЗАмЕчАниЕ. На самом деле, скорость убывания локализованного решения связана со скоростью убывания функции Грина $\widehat{\Gamma}(\cdot)$ (см. следующий пункт) оператора $D F(0)$. Если $\widehat{\Gamma}$ убьвает экспоненциально, то в предположениях леммы 1 локализованные решения убывают экспоненциально.

Лемма 2. Пусть $G=\mathbb{Z}^{d}$ - группа операторов сдвига на $\ell_{\infty}, d\left(g_{1}, g_{2}\right)=\left|g_{1}-g_{2}\right|$. Кроме того, пусть $A, \widetilde{F} \in C^{2}\left(\ell_{\infty}\right)$ - однородные решетки связанных отображений, $A \in \mathscr{L}\left(\ell_{\infty}\right), \widetilde{F}(0)=0$ и $D \widetilde{F}(0)=0$. Предположим, ито $v \in \ell_{1}$. Тогда набор $\left\{v_{k}=v, k=1,2, \ldots\right\}$ является $(\widetilde{F}, G)$-локальным и существуют операторы $\mathscr{A}, B \in \mathscr{L}\left(\ell_{1}\right)$ такие, что $A=\mathscr{A}^{*}$ u $D \widetilde{F}(v)=B^{*}$, а набор операторов $\left\{B_{k}=B, k=1,2, \ldots\right\}$ является $(\mathscr{A}, G)$-локальным.

ДокАЗАТЕЛЬСтво ЛЕммы 1. Укажем предварительные факты и обозначения.

Пусть $(D F(0))^{-1}=\Gamma$, где

$$
(\Gamma u)_{\alpha}=\sum_{\beta \in \mathbb{Z}^{d}} \widehat{\Gamma}(\alpha-\beta) u_{\beta}
$$

с функцией Грина $\widehat{\Gamma}, \widehat{\Gamma}(\alpha) \in \mathscr{L}\left(\mathbb{R}^{p}\right)$. Покажем, почему такое представление возможно. Для простоты рассмотрим случай $p=1$. Так как $D F(0)$ - решетка связанных отображений, $D F(0)=\mathscr{A}^{*}$ для подходящего $\mathscr{A} \in \mathscr{L}\left(\ell_{1}\right)$. По теореме 4 нам достаточно найти представление для $X=\mathscr{A}^{-1}$. Пусть $e_{i} \in \ell_{1}$ - последовательность, где на $i$-м месте стоит 1 а на остальных $0 ; \widehat{e}_{i} \in \ell_{\infty}$ определяется так же, спаривание $(\cdot, \cdot)$ естественное: $\left(\widehat{e}_{i}, e_{j}\right)=\delta_{i, j}$. Операторы из $\ell_{1}$ в $\ell_{1}$ однозначно определяются своими матричными коэффициентами $M_{\alpha, \beta}=\left(\widehat{e}_{\alpha}, X e_{\beta}\right)$, а так как $X$ коммутирует со сдвигами, $M_{\alpha, \beta}=f(\alpha-\beta)$. Переходя к сопряженному оператору, получим искомое представление, причем $K_{\Gamma}=\sum_{\alpha \in \mathbb{Z}^{d}}|\widehat{\Gamma}(\alpha)|<\infty$.

Пусть $\widetilde{F}(u)=F(u)-D F(0) u$ и $\widetilde{f}(x)=f(x)-D f(0) x$ (см. определение 4). Обозначим $r=|I|$.

А) Выберем $\varepsilon_{1}>0$ такое, что если для $\alpha \in I$ вьполняется $\left|u_{\alpha}\right|,\left|v_{\alpha}\right|<\varepsilon_{1}$, то

$$
\left|\widetilde{f}\left(\{u\}_{I}\right)-\widetilde{f}\left(\{v\}_{I}\right)\right|<\left(4 K_{\Gamma} r\right)^{-1} \sum_{\alpha \in I}\left|u_{\alpha}-v_{\alpha}\right| .
$$

Это возможно, так как $\widetilde{f}(0)=0$ и $D \tilde{f}(0)=0$. 
Б) Выберем $0<\varepsilon<\varepsilon_{1}$ такое, что в $(2 \varepsilon)$-окрестности $v$ нет других корней уравнения (6).

Для $m \in \mathbb{N}$ пусть

$$
v_{\alpha}^{(m)}= \begin{cases}v_{\alpha}, & \text { если }|\alpha| \leqslant m, \\ 0, & \text { если }|\alpha|>m .\end{cases}
$$

В) Пусть $s=\max \{|i|: i \in I\}$.

Выберем $M \in \mathbb{N}$ такое, что $\left\|v-v^{(M+s)}\right\|_{\infty}<\varepsilon / 2,\left\|v+\Gamma \widetilde{F}\left(v^{(M+s)}\right)\right\|_{\infty}<\varepsilon / 2$. Напомним, что $\Gamma=(D F(0))^{-1}$. Определим множество

$$
\mathfrak{R}=\left\{\delta \in \ell_{1}: \delta_{\alpha}=v_{\alpha} \text { для }|\alpha| \leqslant M+s \text { и }\left|\delta_{\alpha}\right| \leqslant \varepsilon \text { для }|\alpha|>M+s\right\} .
$$

Рассмотрим отображение $\Phi: \mathfrak{R} \rightarrow \mathfrak{R}$, определенное соотношением

$$
(\Phi(\delta))_{\alpha}=-(\Gamma \widetilde{F}(\delta))_{\alpha} \quad \text { для }|\alpha|>M+r .
$$

Проверим включение $\Phi(\mathfrak{R}) \subset \mathfrak{R}$ :

$$
\|\Phi(u)\|_{1} \leqslant\left(r K_{\Gamma} \sup _{|x|<\|u\|_{1}}|D \tilde{f}(x)|\right)\|u\|_{1}
$$

и

$$
\begin{aligned}
\left|(\Phi(\delta))_{\alpha}\right| & =\left|\sum_{|\beta| \leqslant M} \widehat{\Gamma}(\alpha-\beta) \tilde{f}\left(\left\{S_{\beta} v\right\}_{I}\right)\right|+\left|\sum_{|\beta|>M} \widehat{\Gamma}(\alpha-\beta) \widetilde{f}\left(\left\{S_{\beta} \delta\right\}_{I}\right)\right| \\
& <\frac{1}{2} \varepsilon+K_{\Gamma} \sup _{|\beta|>M} \tilde{f}\left(\left\{S_{\beta} \delta\right\}_{I}\right)<\varepsilon, \quad \delta \in \mathfrak{R}
\end{aligned}
$$

(последние неравенство верно в силу А) и определения $\mathfrak{R}$ ).

$\mathrm{C}$ другой стороны, $\mathfrak{R}$, снабженное метрикой $\left\|\delta_{1}-\delta_{2}\right\|_{1}$, есть замкнутое метрическое пространство и $\Phi-$ сжатие на нем:

$$
\left\|\Phi\left(\delta_{1}\right)-\Phi\left(\delta_{2}\right)\right\|_{1} \leqslant \sum_{\alpha, \beta \in \mathbb{Z}^{d}}|\widehat{\Gamma}(\alpha-\beta)| \cdot\left|\tilde{f}\left(\left\{S_{\beta} \delta_{1}\right\}_{I}\right)-\tilde{f}\left(\left\{S_{\beta} \delta_{2}\right\}_{I}\right)\right| \leqslant \frac{1}{2}\left\|\delta_{1}-\delta_{2}\right\|_{1}
$$

Таким образом, существует единственное $\delta_{0} \in \mathfrak{R}, \delta_{0}=\Phi\left(\delta_{0}\right)$. Мы имеем $v=\delta_{0}$ в силу Б). Лемма 1 доказана.

ДокАЗАТЕЛЬСтво ЛЕммы 2. Для любого $\varepsilon>0$ найдется $M \in \mathbb{N}$ такое, что

$$
\sum_{|\alpha|>M-s}\left|v_{\alpha}\right|<\varepsilon
$$

Пусть $\left|\beta_{j}-\beta_{k}\right|>M$ для $j \neq k$. Тогда

$$
\left\|\widetilde{F}\left(\sum S_{\beta_{j}} v\right)-\sum \widetilde{F}\left(S_{\beta_{j}} v\right)\right\|_{\infty}<2 \varepsilon K_{1}
$$

и

$$
\left\|D \widetilde{F}\left(\sum S_{\beta_{j}} v\right)-\sum D \widetilde{F}\left(S_{\beta_{j}} v\right)\right\|_{\infty}<2 \varepsilon K_{2}
$$

где

$$
K_{1}=\sup _{\left|x_{j}\right| \leqslant\|v\|+\varepsilon}|D \tilde{f}(x)|, \quad K_{2}=\sup _{\left|x_{j}\right| \leqslant\|v\|+\varepsilon}\left|D^{2} \tilde{f}(x)\right| .
$$


Мы доказали, что набор $\left\{v_{k}=v, k=1,2, \ldots\right\}$ является $(\widetilde{F}, G)$-локальным.

Осталось доказать, что $D \widetilde{F}(0)=B^{*}$, и $(\mathscr{A}, G)$-локальность $B$. Имеем

$$
(D \widetilde{F}(v) \xi)_{\alpha}=\sum_{|\beta-\alpha| \leqslant s} \widehat{B}(\alpha, \beta) \xi_{\beta}
$$

и

$$
\sum_{\alpha \in \mathbb{Z}^{d}} \sum_{|\beta-\alpha| \leqslant s}|\widehat{B}(\alpha, \beta)|=K<\infty .
$$

Таким образом, искомое $B \in \mathscr{L}\left(\ell_{1}\right)$ определяется равенством

$$
(B \xi)_{\beta}=\sum_{|\alpha-\beta| \leqslant s} \widehat{B}^{*}(\alpha, \beta) \xi_{\alpha}
$$

Пусть $\Gamma=A^{-1}, T=B \Gamma^{*}$ и $(T u)_{\alpha}=\sum_{\beta} \widehat{T}(\alpha, \beta) u_{\beta}$. Имеем

$$
\sum_{\alpha, \beta \in \mathbb{Z}^{d}}|\widehat{T}(\alpha, \beta)| \leqslant \sum_{\alpha, \beta \in \mathbb{Z}^{d}} \sum_{|\gamma-\alpha| \leqslant s}\left|\widehat{B}^{*}(\gamma, \alpha)\right| \cdot\left|\widehat{\Gamma}^{*}(\beta-\gamma)\right|=K_{T}<\infty .
$$

Осталось убедиться, что $T$ удовлетворяет условиям теоремы 2 . Действительно,

$$
\sum_{j}\left\|\left(S_{\beta_{j}} T S_{\beta_{j}}^{-1}\right) u\right\|_{1} \leqslant K_{T}\|u\|_{1}
$$

если $\left|\beta_{j}-\beta_{k}\right| \geqslant 1$ при $j \neq k$. Мы можем определить $T_{l}$ и $C_{l}$ из теоремы 2 следующим образом:

$$
\left(T_{l} u\right)_{\alpha}= \begin{cases}\sum_{|\beta| \leqslant l} \widehat{T}(\alpha, \beta) u_{\beta}, & \text { если }|\alpha| \leqslant l \\ 0, & \text { если }|\alpha|>l,\end{cases}
$$

и $C_{l}=l+1$.

\section{СПИСОК ЦИТИРОВАННОЙ ЛИТЕРАТУРЫ}

[1] Като Т. Теория возмущений линейных операторов. М.: Мир, 1972.

[2] Kaneko K. (ed. ) Theory and applications of coupled map lattices. New York: Wiley, 1993.

[3] Afraimovich V.S., Chow Shu-Nee, Shen Wenxian. Hyperbolic homoclinic points of $\mathbb{Z}^{d}$-actions in lattice dynamical systems // Internat. J. Bifur. Chaos Appl. Sci. Engrg. 1996. V. 6. №6. P. 1059-1075.

[4] Лерман Л. М., Шильников Л. П. Гомоклинические структуры в бесконечномерных системах // Сиб. матем. ж. 1988. Т. 29. № 3. С. 92-103.

[5] Нитецки 3. Введение в дифференциальную динамику. М.: Мир, 1975.

Нижегородский государственный университет им. Н. И. Лобачевского

Институт прикладной физики РАН, г. Нижний Новгород

Поступило 25.03 .97

E-mail : glebsky@hale.appl.sci-nnov.ru

Исправленный вариант

05.05 .98 